\title{
Exacerbation of Pain by Anxiety Is Associated with Activity in a Hippocampal Network
}

\author{
Alexander Ploghaus, ${ }^{1,2}$ Charvy Narain, ${ }^{1}$ Christian F. Beckmann, ${ }^{1}$ Stuart Clare, ${ }^{1}$ Susanna Bantick, ${ }^{1}$ \\ Richard Wise, ${ }^{1}$ Paul M. Matthews, ${ }^{1}$ J. Nicholas P. Rawlins, ${ }^{2}$ and Irene Tracey ${ }^{1}$ \\ ${ }^{1}$ Oxford Centre for Functional Magnetic Resonance Imaging of the Brain, Department of Clinical Neurology, University of \\ Oxford, John Radcliffe Hospital, Oxford OX3 9DU, United Kingdom, and '2Department of Experimental Psychology, \\ University of Oxford, Oxford OX1 3UD, United Kingdom
}

It is common clinical experience that anxiety about pain can exacerbate the pain sensation. Using event-related functional magnetic resonance imaging (FMRI), we compared activation responses to noxious thermal stimulation while perceived pain intensity was manipulated by changes in either physical intensity or induced anxiety. One visual signal, which reliably predicted noxious stimulation of moderate intensity, came to evoke low anxiety about the impending pain. Another visual signal was followed by the same, moderate-intensity stimulation on most of the trials, but occasionally by discriminably stronger noxious stimuli, and came to evoke higher anxiety. We found that the entorhinal cortex of the hippocampal formation responded differentially to identical noxious stimuli, dependent on whether the perceived pain intensity was enhanced by pain-relevant anxiety. During this emotional pain modulation, entorhinal responses predicted activity in closely connected, affective (perigenual cingulate), and intensity coding (midinsula) areas. Our finding suggests that accurate preparatory information during medical and dental procedures alleviates pain by disengaging the hippocampus. It supports the proposal that during anxiety, the hippocampal formation amplifies aversive events to prime behavioral responses that are adaptive to the worst possible outcome.

Key words: hyperalgesia; hippocampus; classical fear conditioning; anterior cingulate; insula; causal associative learning; medial temporal lobe; surprise; aversive emotional learning; anticipation; functional neuroimaging
The positive relationship between anxiety and pain is a common experience in clinical settings (Sternbach, 1968; Melzack, 1973; Grachev et al., 2001). For example, anxiety levels have been shown to predict pain severity and pain behavior in acute and chronic pain patients (Kain et al., 2000; van den Hout et al., 2001), and anxiety reduction techniques and anxiolytic drugs have been reported to be successful in ameliorating pain associated with medical procedures (Suls and Wan, 1989; Dellemijn and Fields, 1994). Experimental studies have confirmed the enhancing effect of anxiety on pain for different components and measures of pain, e.g., ratings of pain intensity (Al Absi and Rokke, 1991) and unpleasantness (Weisenberg et al., 1984), pain threshold (Rhudy and Meagher, 2000), and pain discrimination (Schumacher and Velden, 1984). Anxiolytic drugs reverse the experimental effect (Gracely et al., 1978; Janssen and Arntz, 1999).

Functional neuroimaging studies have greatly advanced our understanding of the neural mechanisms mediating the emotional consequences of tissue stress (Rainville et al., 1997; Tölle et al., 1999; Casey et al., 2001). In contrast, little is known about the human forebrain mechanisms underlying the reverse causality, i.e., the pathways whereby emotions, particularly anxiety, can

\footnotetext{
Received Aug. 7, 2001; revised Sept. 26, 2001; accepted Oct. 2, 2001.

We thank Michael Fanselow, Gary Van Hoesen, Stuart Derbyshire, and Predrag Petrovic for helpful comments. A.P. holds a Junior Research Fellowship at Merton College (Oxford, UK), and I.T. and the Oxford Centre for Functional Magnetic Resonance Imaging of the Brain are funded by the Medical Research Council (UK).

Correspondence should be addressed to Alexander Ploghaus, Department of Neuroradiology, Massachusetts General Hospital and Harvard Medical School, 13th Street, Building 149, Charlestown, MA 02129. E-mail: ploghaus@nmr.mgh. harvard.edu.

Copyright @ 2001 Society for Neuroscience 0270-6474/01/219896-08\$15.00/0
}

enhance pain sensitivity. In a previous study, we used functional magnetic resonance imaging (FMRI) to reveal distinct neural substrates for pain and its anticipation (Ploghaus et al., 1999). However, this and related experiments (Reiman et al., 1989; Hsieh et al., 1999; Sawamoto et al., 2000; Naliboff et al., 2001) did not assess the effect of anticipation on pain perception. The study by Ploghaus et al. (2000), the first neuroimaging study to demonstrate that brain responses to surprising events are predicted by formal associative learning theory, was designed to exclude the modulatory effect of anxiety on pain. Emotional pain modulation has been studied extensively in experimental animals (Fanselow, 1985; Maier, 1986; Helmstetter, 1992), but there is currently no animal model of anxiety-induced hyperalgesia.

The present study examined the neural mechanism by which anxiety causes hyperalgesia and contrasted it with the process by which enhanced nociceptive stimulation increases pain. We used event-related FMRI (Davis et al., 1998a), and we adapted a differential Pavlovian delay conditioning task to the withinsubject within-session requirements of FMRI. In the task, one visual signal was always followed by the same, lower-temperature nociceptive stimulation (LT) to the left hand. This signal came to evoke low anxiety about the impending pain. Another visual signal was followed by LT on most of the trials, but occasionally by a higher-temperature noxious stimulus (HT). This signal came to evoke higher anxiety. We obtained anxiety and pain ratings for each trial. This task allowed us to assess whether pain ratings and localized brain responses to physically identical noxious stimuli vary as a function of pain-relevant anxiety levels. We hypothesized that anxiety-induced hyperalgesia is associated with activity in a subset of the brain regions that respond to experimental 
Signal condition

Pain condition

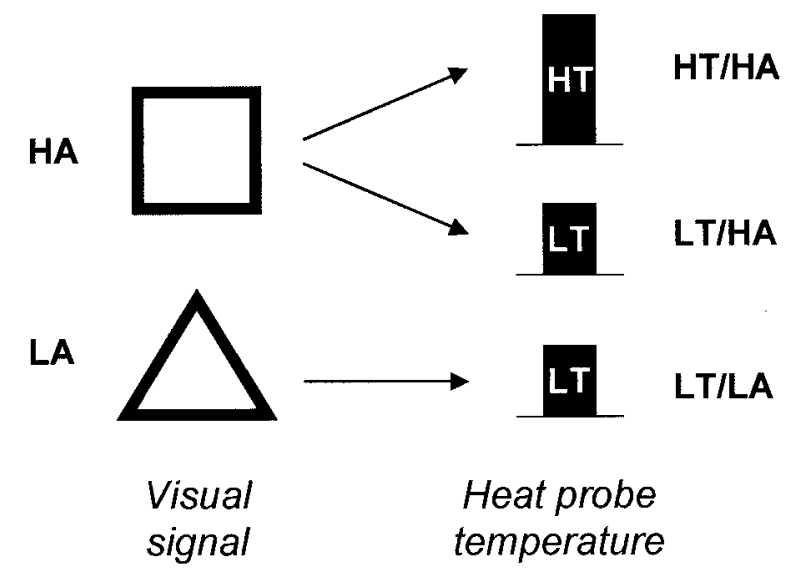

Figure 1. Relevant experimental conditions. Visual signals predicted painful heat stimulation to the back of the left hand. Painful stimulation was delivered either at a lower $(L T)$ or at a discriminably higher $(H T)$ temperature. One visual signal (here: triangle) was consistently followed by LT and came to evoke low anxiety $(L A)$. Another signal (here: square) was followed by LT on most of the trials, but occasionally by HT, and came to evoke higher anxiety $(H A)$. We studied anxiety-induced increases in perceived pain intensity by comparing brain responses to pain in conditions LT/HA and LT/LA. We also assessed temperature-induced increases in perceived pain intensity by comparing brain responses to pain in conditions $\mathrm{HT} / \mathrm{HA}$ and LT/HA.

nociceptive stimulation (for review, see Casey, 1999; Gelnar et al., 1999; Treede et al., 1999; Davis, 2000; Peyron et al., 2000).

\section{MATERIALS AND METHODS}

Subjects and neuroimaging. Eight healthy, right-handed male volunteers with ages ranging from 22 to 40 years participated in the study. All subjects gave informed consent, and the study was approved by the Oxfordshire Committee for Research Ethics. Data were acquired on a 3T whole-body scanner (Siemens, Erlangen, Germany) with a quadrature birdcage head coil. Head movements were restrained with foam pads. In each of 21 contiguous planes, 579 blood-oxygenation level-dependent (BOLD) images were acquired continuously by using multishot echoplanar imaging (EPI) with TE of $30 \mathrm{msec}$, TR of $3 \mathrm{sec}$, flip angle of $90^{\circ}$, in-plane resolution of $3.5 \mathrm{~mm}$, slice thickness of $8 \mathrm{~mm}$, and no slice gap. Slices were prescribed in coronal orientation perpendicular to the anterior commissure-posterior commissure (AC-PC) line and covered the entire brain volume (Tracey et al., 2000). Structural images were obtained with a standard T1-weighted pulse sequence.

Psychological task. Thermal stimuli were applied to the dorsum of the left hand with a $1.5 \times 2 \mathrm{~cm}$ thermal resistor designed and built in-house. Visual stimuli (square, triangle, circle) were presented using prism glasses and a back projection screen at feet level outside the scanner. Subjects were presented with thermal stimuli of different temperatures and $6 \mathrm{sec}$ duration throughout scanner setup and were asked to rate their intensity using a five-button box at their right hand as soon as a five-point Likert scale ( $5=$ "extreme pain") was shown. In this study, we used a sensory scale (pain intensity) rather than an emotive one (pain unpleasantness) because a sensory measure is less prone to bias by the concomitant emotional state of anxiety (Gross and Collins, 1981). The scale was presented for a period of $6 \mathrm{sec}$, starting $12 \mathrm{sec}$ after the offset of the thermal stimulus. It was explained that the rating concerned the sensory intensity, not the unpleasantness of pain (Price, 1988). A temperature rated as "moderate pain" in an adaptive sequence (Gracely and Naliboff, 1996) and confirmed on two retest trials, one of which included an EPI noise sample, was used as the LT. The temperature established for LT was increased by $2.5^{\circ} \mathrm{C}$ to obtain an HT. This substantial increase was chosen to ensure clear discrimination between HT and all other painful stimulations. HT was only presented during the experiment itself.

The experimental paradigm (Fig. 1) used delay-conditioning contigu- ities. In conditioning studies, subjects learn causal relations between stimuli by experience, so instructions are limited to necessary information about the constituent stimuli and required responses. Subjects were instructed that they would see shapes on the screen and would receive heat bursts to the back of the left hand. They were asked to figure out the significance of the shapes and rate pain intensity as practiced during thresholding. Experimental conditions were applied in a pseudorandom sequence within a single imaging session for each subject to avoid confounding effects of repetitive noxious heat stimulation, as revealed by Casey et al. (2001). One visual signal was always followed by the LT. This signal came to evoke low anxiety (LA) about the impending pain. The second visual signal was followed by LT on all but two trials (trials 2 and 4), when it was followed by the higher-temperature pain stimulus (HT). This signal came to elicit higher anxiety (HA) about the impending pain. On additional trials, a third signal was presented alone or in compound with HA or LA and was not followed by thermal stimulation. These trials are irrelevant in the present context but can be used to study the neural substrate of a different process, Pavlovian conditioned inhibition. A crossed design would have included a condition HT/LA, but this is impossible because a signal predicting strong noxious stimulation cannot evoke low anxiety.

The delay between signal onset and onset of thermal stimulation (CS-US interval) was pseudorandomized with a mean of $10.4 \mathrm{sec}$ (range, $6-15 \mathrm{sec})$ to optimize aversive conditioning and minimize inhibition of delay. The intertrial interval was also pseudorandomized with a mean of $58 \mathrm{sec}$ (range, $36-72 \mathrm{sec}$ ) to prevent overshadowing of the signals by temporal cues. After each trial, subjects rated the perceived pain intensity in exactly the same way as practiced during thresholding. The $12 \mathrm{sec}$ delay between trial offset and scale onset was chosen to allow for clear separation of trial- and rating-related hemodynamic responses. Heart rate was recorded from the left index finger throughout the experiment using an MR-compatible pulse oximeter (MR Equipment Corp., Bay Shore, NY).

The experiment was also performed in a separate group of nine subjects outside the scanner. The difference was that this group was instructed to rate anxiety about the impending painful stimulus on a five-point Likert scale ( 5 = "extreme anxiety about impending pain") after the onset of each visual signal, but before the onset of thermal stimulation, using the five-button box. It is important to obtain anxiety and pain ratings in separate groups of subjects, because conscious selfassessment of both processes in the same subject can lead to a hypothesis-driven correlation artifact (Gross and Collins, 1981).

Cardiac data analysis. Heart rate responses during aversive signals consist of a series of functionally distinct components (Obrist, 1981). Because the present study used a range of CS-US intervals $(6-15 \mathrm{sec})$, only the initial $6 \mathrm{sec}$ of every signal presentation contain functionally equivalent heart rate components. Accordingly, cardiac data analysis was restricted to this initial interval. Heart rates were converted into percentage of signal change relative to baseline before signal onset.

Event-related FMRI data analysis. The image analysis package FEAT [Centre for Functional Magnetic Resonance Imaging of the Brain (FMRIB), University of Oxford, UK; www.fmrib.ox.ac.uk/fsl] was used for all data processing except motion correction, which was performed using SPM99 (Wellcome Department of Cognitive Neurology, London, UK). The initial four scans of each data set were discarded because of nonequilibrium magnetization, and independent component analysis was applied to detect and remove artifacts from the FMRI time series (Beckmann et al., 2000; Brown et al., 2001). The data were specifically examined for medial temporal lobe susceptibility artifacts by looking at the animated residual time series (Büchel et al., 1998). This procedure revealed no event-related changes in susceptibility. Head motion was corrected, and the data were smoothed in the spatial domain with a three-dimensional $8 \mathrm{~mm}$ (full width at half maximum) isotropic Gaussian kernel. All volumes were scaled by a single factor to obtain the same grand mean across subjects, and the data were filtered in the temporal domain using a nonlinear high-pass filter with a cutoff period of $150 \mathrm{sec}$.

Statistical analysis was based on a least-squares estimation using a general linear model approach (Friston et al., 1995) with nonparametric local autocorrelation correction implemented in FILM (FMRIB, University of Oxford; Woolrich et al., 2000). Boxcar reference functions modeling separately all task components (types of visual signals, types of conditional pain stimuli) as main effects as well as interactions with time were convolved with a $\gamma$-variate model of the hemodynamic response (Cohen, 1997) and its first derivative with respect to time (Josephs et al., 1997). Visual and pain stimuli constituting the first presentation of each 


\begin{tabular}{|c|c|c|c|c|c|c|c|}
\hline \multirow[b]{2}{*}{ Brain region (Brodmann area) } & \multirow[b]{2}{*}{ Side } & \multicolumn{3}{|c|}{$\underline{\text { Talairach coordinates }}$} & \multicolumn{2}{|c|}{$Z$ value } & \multirow{2}{*}{$\begin{array}{l}Z \text { threshold } \\
p=0.05, \text { cor }\end{array}$} \\
\hline & & $X$ & $Y$ & $Z$ & Mean & Max. & \\
\hline \multicolumn{8}{|l|}{ Pain modulation by anxiety } \\
\hline Entorhinal cortex (28) & $\mathrm{L}$ & -22 & -17 & -26 & 3.57 & 4.61 & 3.44 \\
\hline \multirow[t]{2}{*}{ Mid-insula/parainsular cortex $(52)^{*}$} & $\mathrm{R}$ & 44 & -10 & 3 & 3.76 & 4.98 & 3.41 \\
\hline & $\mathrm{L}$ & -53 & -11 & 5 & 3.90 & 5.96 & 3.41 \\
\hline Perigenual cingulate $(24 / 32)^{*}$ & $\mathrm{~L} / \mathrm{R}$ & 1 & 37 & 9 & 3.88 & 5.96 & 3.41 \\
\hline \multicolumn{8}{|l|}{ Pain modulation by temperature } \\
\hline \multirow[t]{2}{*}{ Hippocampus } & $\mathrm{L}$ & -18 & -24 & -13 & 3.46 & 4.30 & 3.34 \\
\hline & $\mathrm{R}$ & 21 & -21 & -14 & 3.78 & 4.82 & 3.34 \\
\hline Orbitofrontal cortex (11) & $\mathrm{L} / \mathrm{R}$ & -1 & 58 & -13 & 3.46 & 4.33 & 3.34 \\
\hline \multirow[t]{2}{*}{$\mathrm{SI} / \mathrm{MI}$} & $\mathrm{L}$ & -52 & -13 & 41 & 3.48 & 4.26 & 3.34 \\
\hline & $\mathrm{R}$ & 42 & -14 & 48 & 3.77 & 5.81 & 3.34 \\
\hline \multirow[t]{2}{*}{ Posterior insula } & $\mathrm{L}$ & -39 & -19 & 15 & 3.56 & 4.39 & 3.34 \\
\hline & $\mathrm{R}$ & 37 & -19 & 14 & 3.60 & 4.63 & 3.34 \\
\hline Thalamus & $\mathrm{L} / \mathrm{R}$ & -1 & -9 & 2 & 3.75 & 4.90 & 3.34 \\
\hline Midcingulate $\left(24^{\prime} / 32^{\prime}\right)$ & $\mathrm{L} / \mathrm{R}$ & 1 & -11 & 46 & 4.02 & 6.20 & 3.34 \\
\hline
\end{tabular}

$p=0.001 ; Z=3.09$.

*Area shows activity significantly correlated with the entorhinal FMRI signal during LT/HA and LT/LA $(p<0.001)$.

trial type were entered separately as regressors of no interest, because their neural representations had not been modified by experience of the experimental contingencies. Linear contrasts between parameter estimates for conditions LT/HA and LT/LA, as well as HT/HA and LT/HA, resulted in mean difference images for each subject. These images were warped into Talairach and Tournoux (1988) stereotaxic space using transformations generated by the two-level, 12-parameter affine registration method implemented in FLIRT (FMRIB, University of Oxford; Jenkinson and Smith, 2001).

Our hypothesis specified our region of interest (ROI) as the pain matrix, and activations were considered to satisfy this criterion if their Talairach coordinates were located in one of the component structures of the pain matrix as tabulated in Peyron et al. (2000). Two SPM thresholding conventions for ROI-based fixed-effects analysis have evolved, (1) $p=0.001$, uncorrected (Elliott et al., 2000), and (2) $p=0.05$, corrected for multiple comparisons given the ROI and the smoothness of the underlying $Z$ statistic image (Büchel et al., 1999). We adopted method (1), but also report in Table 1 the $Z$ thresholds derived by method (2), where the ROI was defined by an average pain activation obtained from an independent data set (Ploghaus et al., 2000). All activations reported here are revealed by both methods. Event-related FMRI is insensitive to stimulus-induced motion (Birn et al., 1999), but we also directly examined the response peak latencies of significant activations. The earliest response peaks were found $\sim 7 \mathrm{sec}$ after pain onset, which renders stimulus-correlated motion unlikely (Birn et al., 1999).

We also examined the potential input-output pathways of regions activated during anxiety-induced pain modulation. For this purpose, we set individual subjects' FMRI signal values for the activated region to the time course average except for the time points associated with the response to LT/HA and LT/LA (see Fig. $4 B$ ), normalized the function, and correlated it with the FMRI time course at every other voxel.

\section{RESULTS}

\section{Behavioral results}

Ratings of pain-related anxiety before the onset of LT were significantly higher for HA than LA (Wilcoxon signed rank test, two-tailed, $Z=2.67 ; p<0.05)$. Anxiety scores were $3.73 \pm 1.11$ (mean $\pm \mathrm{SD}$ ) and $2.28 \pm 0.91$ for HA and LA, respectively (Fig. $2 A$ ). Heart rate (Fig. $2 B$ ) was significantly higher during presentation of LA than HA (Wilcoxon signed rank test, two-tailed, $Z=$ 2.29 ; $p<0.05$ ). Presentation of LA resulted in heart rate acceleration relative to baseline (percentage of change, $2.05 \pm 4.38$ ), whereas heart rate deceleration was found during presentation of HA (percentage of change, $-0.35 \pm 4.58$ ). The painful stimulus
LT was rated as significantly more intense when signaled by HA than LA (Wilcoxon signed rank test, two-tailed, $Z=2.37 ; p<$ $0.05)$. Scores of perceived pain intensity were $1.95 \pm 0.79$ and $2.33 \pm 0.88$ for $\mathrm{LT} / \mathrm{LA}$ and $\mathrm{LT} / \mathrm{HA}$, respectively (Fig. 2C). Ratings of pain intensity were significantly higher during presentation of HT/HA $(3.19 \pm 0.54)$ than LT/HA (Wilcoxon signed rank test, two-tailed, $Z=2.52 ; p<0.05)$.

Ratings of anxiety elicited by signals HA and LA were tested for linear and quadratic trends over time using repeated measures ANOVA. Anxiety associated with LA did not change after the first LA trial, and anxiety associated with HA did not change after the second HA trial. Therefore, within-subject variability in pain ratings and BOLD responses after these trials cannot be accounted for by changes in anxiety, and hence forms part of the error variance. Postexperimental interview confirmed clear intensity discrimination in all subjects of the two HT/HA trials and the other trials involving HA, thus precluding the possibility that the higher pain intensity rating of LT/HA relative to LT/LA was simply because of generalization from HT/HA.

\section{FMRI results}

Conditions HT/HA and LT/HA were equated for anxiety and differed in the intensity of thermal stimulation, whereas conditions LT/HA and LT/LA were equated for stimulation intensity and differed in pain-related anxiety. Thus, we compared hemodynamic responses to pain in conditions $\mathrm{HT} / \mathrm{HA}$ and LT/HA to reveal regional activation associated with temperature-related changes in perceived pain intensity. Crucially, we also compared hemodynamic responses to pain in conditions LT/HA and LT/LA to reveal regional activation associated with anxietyrelated changes in perceived pain intensity.

\section{Temperature-induced pain modulation}

Comparison of conditions HT/HA and LT/HA revealed bilateral activation in primary somatosensory-motor cortex (SI-MI), midcingulate cortex, orbitofrontal cortex, thalamus, hippocampus proper, and posterior insula around the superior marginal sulcus (Fig. 3A). Talairach coordinates and $Z$ values associated with these activations are shown in Table 1. Hippocampal time courses 
A

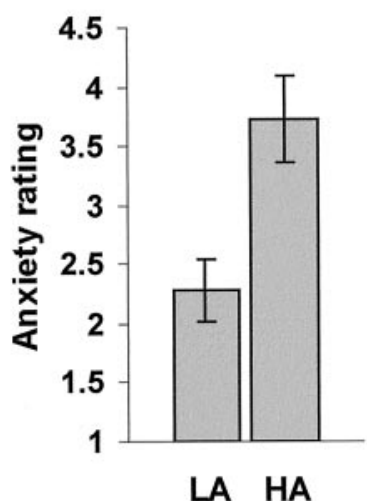

LA HA
B

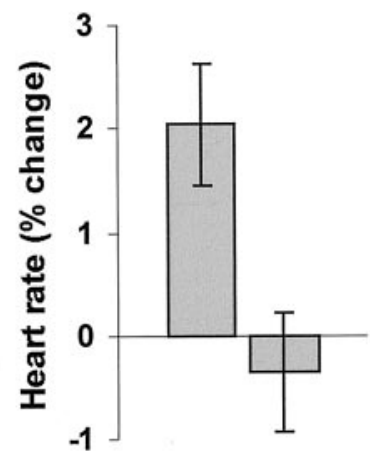

LA HA

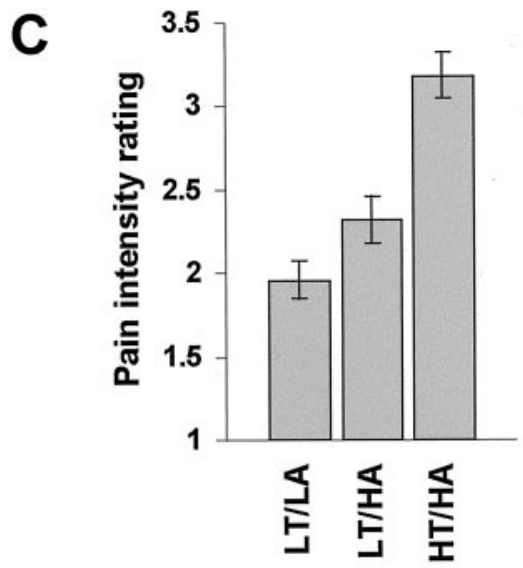

Figure 2. Behavioral results. A, Ratings of anxiety during presentation of signals HA and LA before the onset of pain LT (bench control group, mean \pm one SEM). $B$, Heart rate changes during presentation of signals HA and LA (scanner group, mean \pm one SEM). C, Ratings of perceived pain intensity in conditions LT/LA, LT/HA, and HT/HA (scanner group, mean \pm one SEM).

of FMRI signal for this contrast are shown in Figure $4 A$. These findings are consistent with the results of neuroimaging studies correlating brain activation with perceived pain intensity (Derbyshire et al., 1997; Porro et al., 1998; Coghill et al., 1999, 2001) and demonstrations of orbitofrontal activity during pain (for review, see Petrovic et al., 2000) and abstract punishment (O’Doherty et al., 2001).

\section{Anxiety-induced pain modulation}

Comparison of conditions LT/HA and LT/LA revealed significant activation in the left parahippocampal gyrus (Fig. 3B, Table $1)$. MR volumetric analysis showed that the activation area corresponded to the entorhinal cortex of the hippocampal formation (Insausti et al., 1998). Time courses of FMRI signal in the left entorhinal cortex (Fig. 4B) revealed a positive hemodynamic response in condition LT/HA and a negative hemodynamic response in condition LT/LA. However, the left entorhinal activation found in comparison LT/HA versus LT/LA cannot be accounted for by deactivation in condition LT/LA because the region also activated significantly in the comparison LT/HA versus baseline (maximum $Z=3.96$, mean $Z=3.40, p<0.001$ ).

A
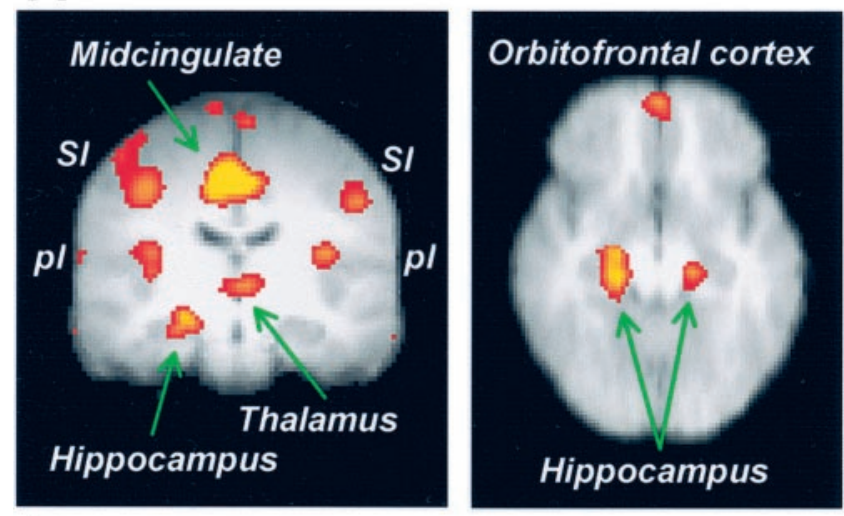

B
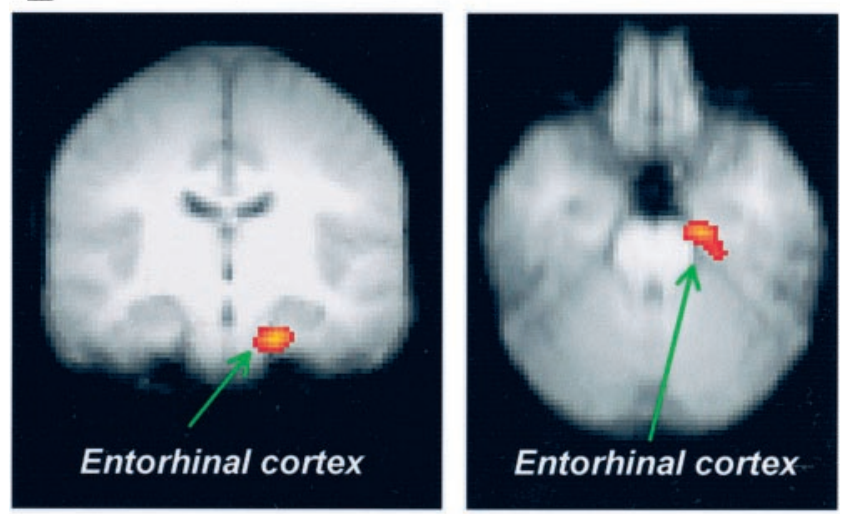

C
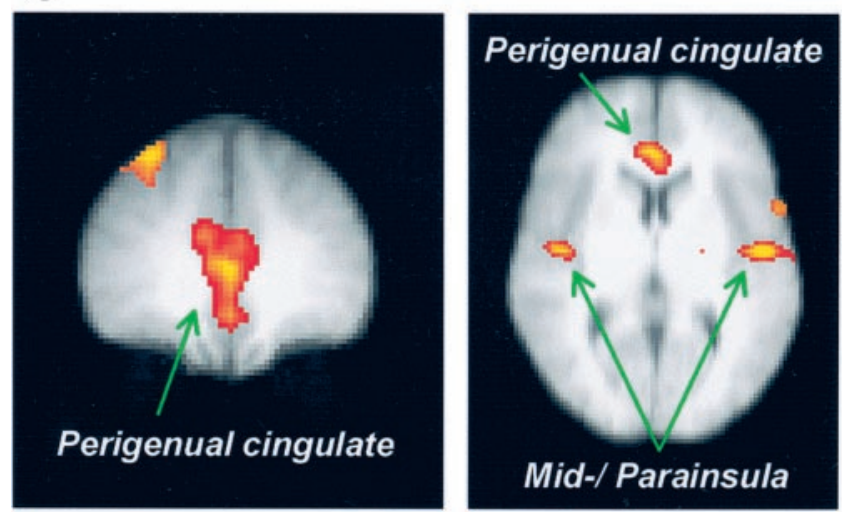

Figure 3. Group $Z$ value maps thresholded at $p<0.001$ and superimposed on an average anatomical MRI of participating subjects in Talairach space (radiological convention). $A$, Significant activations associated with temperature-related increases in perceived pain (HT/HA vs LT/HA). The coronal view (left, $y=-16$ ) shows activations bilaterally in primary somatosensory cortex $(S I)$, dorsal margin of the posterior insula $(p I)$, thalamus, midcingulate cortex, and in the right hippocampus. The horizontal view (right, $z=-14$ ) depicts bilateral hippocampus as well as orbitofrontal cortex. $B$, Anxiety-related increases in perceived pain (LT/HA vs LT/LA) are associated with significant activation in the left entorhinal cortex. The activation area is shown in coronal (left, $y=-16)$ and horizontal (right, $z=-26$ ) view. $C$, Areas showing activity significantly correlated with the entorhinal FMRI signal during pain modulation by anxiety (LT/HA and LT/LA). The coronal view (left, $y=36)$ and the horizontal view (right, $z=6$ ) show activation in the perigenual cingulate cortex, and the horizontal view depicts bilateral activity in the mid-insular and parainsular cortices. 
A

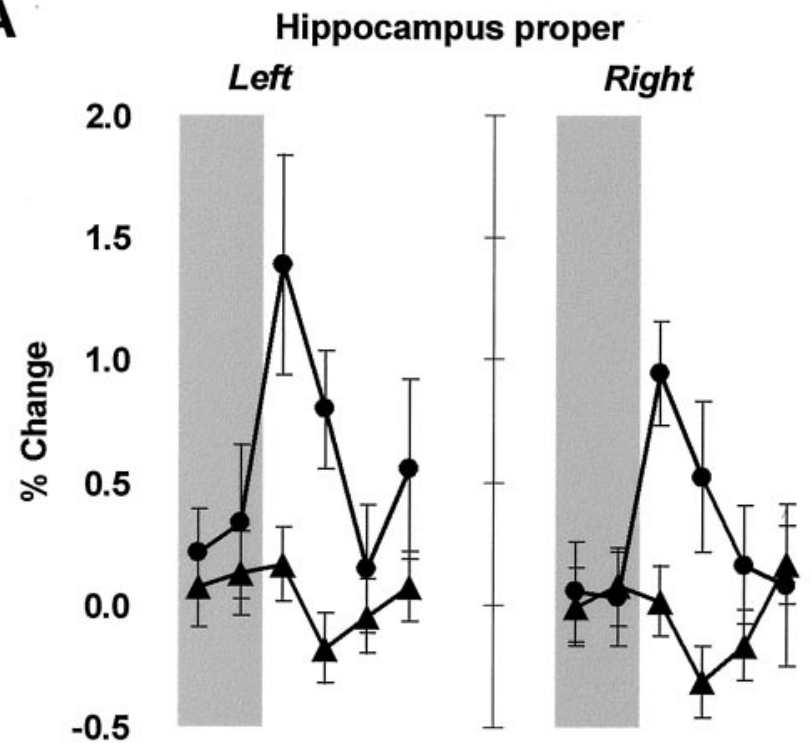

B

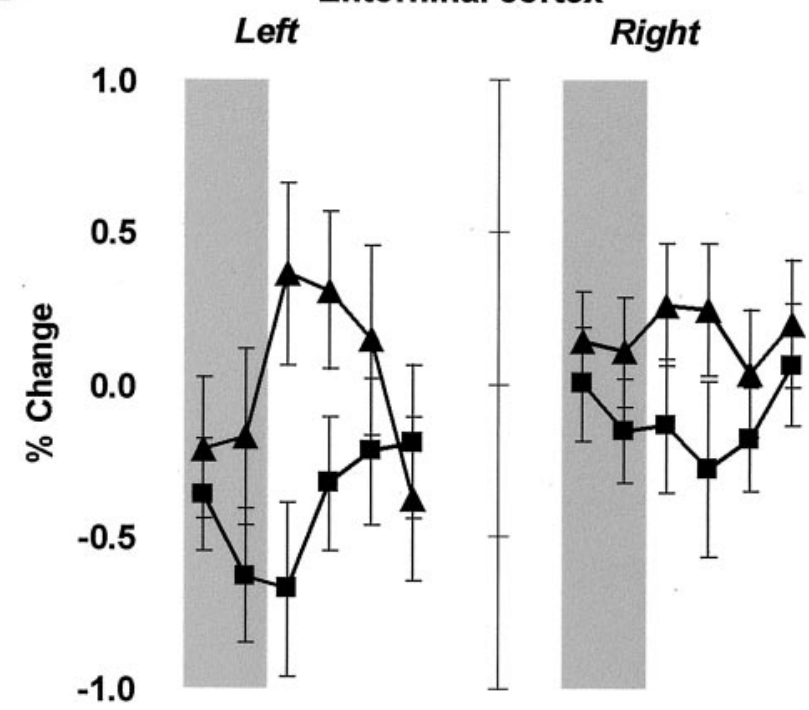

Figure 4. Significantly different hemodynamic responses in the hippocampus proper and entorhinal cortex (group mean \pm one SEM). Regional time courses of FMRI signal represent percentage of change from the rest period preceding each trial, averaged across trials and subjects. The period of painful stimulation is shaded. $A$, The hippocampus proper was significantly activated bilaterally during $\mathrm{HT} / \mathrm{HA}(\bullet)$ relative to LT/HA $(\mathbf{\Delta})$. B, The left entorhinal cortex was significantly activated during LT/HA $(\mathbf{\Delta})$ relative to LT/LA (ם), as well as relative to baseline. The right entorhinal cortex shows similar, but smaller responses, which is consistent with observations of left-lateralized processing of explicit aversive conditioning in the medial temporal lobes.

The right entorhinal cortex showed similar, but smaller responses (Fig. 4B).

There was a trend toward a positive association between individual subjects' mean difference in pain ratings between condition LT/HA and LT/LA, and their mean difference in entorhinal hemodynamic responses (average of volumes 3-5 after pain onset) in these conditions (Pearson $r=0.59, p=0.058$ ). A correlation analysis was performed to obtain some preliminary evi- dence of the afferent or efferent pathways involved. Individual subjects' time courses of FMRI signal in the entorhinal cortex during conditions LT/HA and LT/LA correlated significantly with time courses in the perigenual cingulate and in the midinsula around the inferior marginal sulcus (Fig. 3C, Table 1).

\section{DISCUSSION}

The present study shows that pain-related anxiety can increase perceived pain intensity. Event-related FMRI revealed that pain modulation by anxiety is associated with activation changes in the entorhinal cortex of the hippocampal formation. The entorhinal hemodynamic response was significant both relative to a lowanxiety control condition and relative to baseline, and was predictive of activity in the perigenual cingulate and mid-insula. Pain modulation by temperature also activated the hippocampal formation, but the hemodynamic response originated in the more dorsal region of the hippocampus proper.

\section{Pain processing in the hippocampal formation}

Melzack and Casey (1968) proposed that the hippocampus and associated cortices participate in mediating the aversive drive and affect characteristic of pain. Subsequent studies confirmed a hippocampal role in pain processing (Lathe, 2001) using extracellular recordings (Zheng and Khanna, 1999), intracellular recordings (Wei et al., 2000), hippocampal EEG (Archer and Roth, 1997), in vivo microdialysis (Ceccarelli et al., 1999), immediateearly gene expression (Pearse et al., 2001), long-term potentiation (Wei et al., 2000), and functional neuroimaging (Hsieh et al., 1995; Derbyshire et al., 1997; Becerra et al., 1999; Casey, 1999; Peyron et al., 1999; Schneider et al., 2001). Wei et al. (2000) demonstrated that the amplitude of EPSPs in hippocampal CA1 pyramidal cells is positively related to the intensity of nociceptive stimulation. This is consistent with our observation that hippocampus proper activity varies as a function of the physical intensity of noxious stimulation.

The aforementioned studies cannot rule out the possibility that hippocampal activation during pain signifies processes that are not pain-specific, e.g., memory encoding and retrieval or contextual conditioning. However, stimulation and lesion studies confirm that pain processing is a primary function of the hippocampus. Prado and Roberts (1985) showed that the dorsal hippocampus is one of the two brain regions where electrical stimulation alters nociception, but where, crucially, the electrical stimulation itself is not perceived as aversive. Sinha et al. (1999) found that stimulation of the hippocampus disrupts the jawopening reflex evoked by phasic tooth pulp pain. McKenna and Melzack (1992) demonstrated that injection of the local anesthetic lidocaine into the dentate gyrus reduces pain sensitivity in rats. Interestingly, the amnesic patient H.M., who underwent bilateral hippocampectomy to alleviate severe epilepsy, had marked impairments in pain perception (Hebben et al., 1985).

Neuropharmacological findings single out the entorhinal cortex as one possible source of pain modulation in the hippocampal formation (Hurd, 1996; Fiore et al., 1999). In particular, there appears to be an association between unconditioned pain modulation and expression of the immediate-early gene c-fos in the entorhinal cortex. Pain of moderate intensity leads to hyperalgesia (King et al., 1996) and increased entorhinal c-fos expression (Funahashi et al., 1999), whereas strong pain leads to hypoalgesia (King et al., 1996) and decreased entorhinal c-fos expression (Funahashi et al., 1999). Our finding provides an experimental demonstration of the apparent association between entorhinal 
activity and pain modulation and shows that the entorhinal cortex mediates not only unconditioned, but also conditioned hyperalgesia. The left entorhinal cortex showed a stronger response than the right one, which is consistent with the preferential engagement of left medial temporal lobe structures during explicit conditioning tasks (Morris et al., 1998; Chun and Phelps, 1999).

\section{Entorhinal interactions with cingulate and insula}

Our preliminary evidence of entorhinal interactions with the perigenual cingulate and mid-insula is consistent with direct projections between these regions. Generally, the pattern of cortical connectivity of the entorhinal cortex resembles that of the amygdala (Van Hoesen, 1995). The entorhinal cortex maintains a substantial reciprocal connection with the perigenual cingulate, which is much stronger than its connection with the midcingulate (Insausti et al., 1987; Amaral and Insausti, 1990). Within the insular region, the entorhinal cortex projects most heavily to the parainsular cortex (Insausti et al., 1987), which forms part of the activation area we identified in the mid-insula. The mid-insula, in turn, has stronger connections with the perigenual cingulate than the anterior insula (Vogt et al., 1996).

The perigenual cingulate has been termed the affective subdivision of the anterior cingulate cortex (Devinsky et al., 1995; Bush et al., 2000) and has a demonstrated role in anxiety. It is activated by aversive conditioned stimuli (Büchel et al., 1999; Ploghaus et al., 1999), and lesions attenuate endocrine and autonomic (Devinsky et al., 1995) as well as avoidance (Johansen et al., 2001) responses to such stimuli. The perigenual cingulate also responds to symptom provocation in patients with anxiety disorders (Rauch et al., 1995). The correlated activity we observed in this region during pain modulation by anxiety may therefore reflect the changes in anxiety itself.

The mid-posterior insula mediates thermosensitivity (Craig et al., 2000), e.g., the sensory dimension of thermal pain (Peyron et al., 1999; Hofbauer et al., 2001). The present study found that objective increases in noxious thermal stimulation activated the posterior insula, whereas correlated activity during anxietyinduced pain modulation occurred in the mid-insula. Perceptual modulation of an innocuous stimulus by uncertain expectation of pain activates the posterior insula (Sawamoto et al., 2000). Painful stimulation activates both areas (Craig et al., 1996; Davis et al., 1998b; Iadarola et al., 1998; Treede et al., 2000) as a function of perceived pain intensity (Derbyshire et al., 1997; Coghill et al., 1999, 2001; Casey et al., 2001). Our finding may therefore suggest that the entorhinal cortex mediates anxiety-induced hyperalgesia by influencing intensity coding in the mid-insula. This would be consistent with the Gray-McNaughton theory of hippocampal function, which predicts that during anxiety, the hippocampal formation increases pain by sending amplifying signals to the neural representation of the pain stimulus.

\section{Gray-McNaughton theory and pain modulation}

The Gray-McNaughton theory (Gray and McNaughton, 2000) proposes that the hippocampal formation responds to aversive events (e.g., pain) whenever they form part of a behavioral conflict. It resolves the conflict by sending amplification signals to the neural representation of the aversive event, thereby biasing the organism toward a behavior that is adaptive to the worst possible outcome. This process is accompanied by anxiety. A role for the entorhinal cortex in detecting conflict has also been proposed in memory models of the hippocampus (Lavenex and Amaral, 2000; Witter et al., 2000).
Applied to the present experiment, behavioral conflict may arise in condition LT/HA because signal HA is not a reliable predictor of pain intensity. Therefore, without intervention of the hippocampal formation, responses adaptive to different levels of pain would compete until asymptotic pain intensity becomes clear (at pain offset at the latest). The hippocampus may resolve this conflict earlier by amplifying pain intensity, thereby giving priority to responses adaptive to the more intense pain, HT. Signal LA, in contrast, reliably predicts asymptotic pain intensity so that no behavioral conflict arises. The Gray-McNaughton theory therefore predicts that during $\mathrm{LT} / \mathrm{HA}$ relative to $\mathrm{LT} / \mathrm{LA}$, there should be (1) higher anxiety, (2) stronger pain, and increased activity in (3) the hippocampal formation and (4) neural representations of pain and anxiety. We found supporting evidence for the first three predictions, and preliminary evidence consistent with prediction (4).

Behavioral studies have established that during medical and dental procedures, pain is alleviated by accurate preparatory information or, in other words, by reliable prediction (for review, see Miller, 1981; Suls and Wan, 1989). Our finding suggests that this intervention is effective by disengaging the hippocampal formation. Heart rate changes observed in the present study may support this interpretation. Subjects showed heart rate decreases during HA and increases during LA. The defense cascade model (Lang et al., 2000) suggests that with decreasing defensive distance (i.e., increasing certainty about the impending aversive event), heart rate first decreases, and then increases relative to baseline. Similarly, Obrist (1981) reports that behavioral conflict leads to cardiodeceleration, whereas reliable prediction of an aversive outcome results in cardioacceleration.

Anxiety has been defined as "... apprehension, tension, or uneasiness that stems from the anticipation of danger. The manifestations of anxiety ... include motor tension, autonomic hyperactivity, apprehensive expectation, and vigilance and scanning. . " (American Psychiatric Association, 1987, p. 392). Thus, anxiety is defined by the shaping of cognitive and other processes toward detecting and eliminating threat (Keogh et al., 2001), and therefore provides a more specific explanation of our results than its component processes in their general form (e.g., "expectation"). In addition, the hypoalgesic effect of anxiolytic drugs makes it appear unlikely that, in the present study, any of the component processes make an important hyperalgesic contribution independently of the state of anxiety.

\section{Conclusion}

The present study showed that anxiety-induced hyperalgesia is associated with activation in the entorhinal cortex of the hippocampal formation. This is consistent with the Gray-McNaughton theory, which proposes that during anxiety, the hippocampal formation increases the valence of aversive events to prime behavioral responses adaptive to the worst possible outcome. Our observation helps to interpret anatomical, neuropharmacological, and electrophysiological evidence implicating the hippocampal formation in pain modulation. Our finding suggests that accurate preparatory information during medical and dental procedures alleviates pain by disengaging the hippocampal formation. Searching for interventions to specifically modulate hippocampal activation offers an approach to identifying new treatments for procedural pain and some forms of chronic pain.

\section{REFERENCES}

Al Absi M, Rokke PD (1991) Can anxiety help us tolerate pain? Pain 46:43-51. 
Amaral DG, Insausti R (1990) Hippocampal formation. In: The human nervous system (Paxinos G, ed), pp 711-755. San Diego, CA: Academic.

American Psychiatric Association (1987) Diagnostic and statistical manual of mental disorders (Ed 3, revised). Washington: APA.

Archer DP, Roth SH (1997) Pharmacodynamics of thiopentone: nocifensive reflex threshold changes correlate with hippocampal electroencephalography. Br J Anaesth 79:744-749.

Becerra LR, Breiter HC, Stojanovic M, Fishman S, Edwards A, Comite AR, Gonzales RG, Borsook D (1999) Human brain activation under controlled thermal stimulation and habituation to noxious heat: an fMRI study. Magn Reson Med 41:1044-1057.

Beckmann C, Noble J, Smith S (2000) Artefact detection in FMRI data using independent component analysis. NeuroImage 11:614.

Birn RM, Bandettini PA, Cox RW, Shaker R (1999) Event-related fMRI of tasks involving brief motion. Hum Brain Mapp 7:106-114.

Brown GD, Yamada S, Sejnowski TJ (2001) Independent component analysis at the neural cocktail party. Trends Neurosci 24:54-63.

Büchel C, Morris J, Dolan RJ, Friston KJ (1998) Brain systems mediating aversive conditioning: an event-related fMRI study. Neuron 20:947-957.

Büchel C, Dolan RJ, Armony JL, Friston KJ (1999) Amygdalahippocampal involvement in human aversive trace conditioning revealed through event-related functional magnetic resonance imaging. J Neurosci 19:10869-10876.

Bush G, Luu P, Posner MI (2000) Cognitive and emotional influences in anterior cingulate cortex. Trends Cogn Sci 4:215-222.

Casey KL (1999) Forebrain mechanisms of nociception and pain: analysis through imaging. Proc Natl Acad Sci USA 96:7668-7674.

Casey KL, Morrow TJ, Lorenz J, Minoshima S (2001) Temporal and spatial dynamics of human forebrain activity during heat pain: analysis by positron emission tomography. J Neurophysiol 85:951-959.

Ceccarelli I, Casamenti F, Massafra C, Pepeu G, Scali C, Aloisi AM (1999) Effects of novelty and pain on behavior and hippocampal extracellular ACh levels in male and female rats. Brain Res 815:169-176.

Chun MM, Phelps EA (1999) Memory deficits for implicit contextual information in amnesic subjects with hippocampal damage. Nat Neurosci 2:844-847.

Coghill RC, Sang CN, Maisog JM, Iadarola MJ (1999) Pain intensity processing within the human brain: a bilateral, distributed mechanism. J Neurophysiol 82:1934-1943.

Coghill RC, Gilron I, Iadarola MJ (2001) Hemispheric lateralization of somatosensory processing. J Neurophysiol 85:2602-2612.

Cohen MS (1997) Parametric analysis of fMRI data using linear systems methods. NeuroImage 6:93-103.

Craig AD, Reiman EM, Evans A, Bushnell MC (1996) Functional imaging of an illusion of pain. Nature 384:258-260.

Craig AD, Chen K, Bandy D, Reiman EM (2000) Thermosensory activation of insular cortex. Nat Neurosci 3:184-190.

Davis KD (2000) Studies of pain using functional magnetic resonance imaging. In: Pain imaging. Progress in pain research and management, Vol 18 (Casey KL, Bushnell MC, eds), pp 195-210. Seattle: IASP.

Davis KD, Kwan CL, Crawley AP, Mikulis DJ (1998a) Event-related fMRI of pain: entering a new era in imaging pain. NeuroReport 9:3019-3023.

Davis KD, Kwan CL, Crawley AP, Mikulis DJ (1998b) Functional MRI study of thalamic and cortical activations evoked by cutaneous heat, cold, and tactile stimuli. J Neurophysiol 80:1533-1546.

Dellemijn PL, Fields HL (1994) Do benzodiazepines have a role in chronic pain management? Pain 57:137-152.

Derbyshire SWG, Jones AKP, Gyulai F, Clark S, Townsend D, Firestone LL (1997) Pain processing during three levels of noxious stimulation produces differential patterns of central activity. Pain 73:431-445.

Devinsky O, Morrell MJ, Vogt BA (1995) Contributions of anterior cingulate cortex to behaviour. Brain 118:279-306.

Elliott R, Friston KJ, Dolan RJ (2000) Dissociable neural responses in human reward systems. J Neurosci 20:6159-6165.

Fanselow MS (1985) Odors released by stressed rats produce opioid analgesia in unstressed rats. Behav Neurosci 99:589-592.

Fiore M, Talamini L, Angelucci F, Koch T, Aloe L, Korf J (1999) Prenatal methylazoxymethanol acetate alters behavior and brain NGF levels in young rats: a possible correlation with the development of schizophrenia-like deficits. Neuropharmacology 38:857-869.

Friston K, Holmes AP, Worsley K, Poline JB, Frith C, Frackowiak RSJ (1995) Statistical parametric maps in functional imaging: a general linear approach. Hum Brain Mapp 2:189-210.

Funahashi M, He YF, Sugimoto T, Matsuo R (1999) Noxious tooth pulp stimulation suppresses c-fos expression in the rat hippocampal formation. Brain Res 827:215-220.

Gelnar PA, Krauss BR, Sheehe PR, Szeverenyi NM, Apkarian AV (1999) A comparative fMRI study of cortical representations for thermal painful, vibrotactile, and motor performance tasks. NeuroImage 10:460-482.

Gracely RH, McGrath P, Dubner R (1978) Validity and sensitivity of ratio scales of sensory and affective verbal pain descriptors: manipulation of affect by diazepam. Pain 5:19-29.

Gracely RH, Naliboff BD (1996) Measurement of pain sensation. In: Pain and touch. Handbook of perception and cognition (Kruger L, ed), pp 243-313. San Diego, CA: Academic.

Grachev ID, Fredickson BE, Apkarian AV (2001) Dissociating anxiety from pain: mapping the neuronal marker $N$-acetyl aspartate to perception distinguishes closely interrelated characteristics of chronic pain. Mol Psychiatry 6:256-260.

Gray JA, McNaughton N (2000) The neuropsychology of anxiety. Oxford: Oxford UP.

Gross RT, Collins FL (1981) On the relationship between anxiety and pain: a methodological confounding. Clin Psychol Rev 1:375-386.

Hebben N, Corkin S, Eichenbaum H, Shedlack K (1985) Diminished ability to interpret and report internal states after bilateral medial temporal resection: case H.M. Behav Neurosci 99:1031-1039.

Helmstetter FJ (1992) The amygdala is essential for the expression of conditioned hypoalgesia. Behav Neurosci 106:518-528.

Hofbauer RK, Rainville P, Duncan GH, Bushnell MC (2001) Cortical representation of the sensory dimension of pain. J Neurophysiol 86:402-411.

Hsieh JC, Ståhle-Bäckdahl M, Hägermark Ö, Stone-Elander S, Rosenquist G, Ingvar M (1995) Traumatic nociceptive pain activates the hypothalamus and the periaqueductal gray: a positron emission tomography study. Pain 64:303-314.

Hsieh JC, Stone-Elander S, Ingvar M (1999) Anticipatory coping of pain expressed in the human anterior cingulate cortex: a positron emission tomography study. Neurosci Lett 262:61-64.

Hurd YL (1996) Differential messenger RNA expression of prodynorphin and proenkephalin in the human brain. Neuroscience 72:767-783.

Iadarola MJ, Berman KF, Zeffiro TA, Byas-Smith MG, Gracely RH, Max MB, Bennett GJ (1998) Neural activation during acute capsaicinevoked pain and allodynia assessed with PET. Brain 121:931-947.

Insausti R, Amaral DG, Cowan WM (1987) The entorhinal cortex of the monkey: II. Cortical afferents. J Comp Neurol 264:356-395.

Insausti R, Juottonen K, Soininen H, Insausti AM, Partanen K, Vainio P, Laakso MP, Pitkänen A (1998) MR volumetric analysis of the human entorhinal, perirhinal, and temporopolar cortices. Am J Neuroradiol 19:659-671.

Janssen SA, Arntz A (1999) No interactive effect of naltrexone and benzodiazepines on pain during phobic fear. Behav Res Ther 37:77-86.

Jenkinson M, Smith SM (2001) A global optimisation method for robust affine registration of brain images. Med Image Anal 5:143-156.

Johansen JP, Fields HL, Manning BH (2001) The affective component of pain in rodents: direct evidence for a contribution of the anterior cingulate cortex. Proc Natl Acad Sci USA 98:8077-8082.

Josephs O, Turner R, Friston KJ (1997) Event-related fMRI. Hum Brain Mapp 5:243-248.

Kain ZN, Sevarino F, Alexander GM, Pincus S, Mayes LC (2000) Preoperative anxiety and postoperative pain in women undergoing hysterectomy-a repeated measures design. J Psychosom Res 49:417-422.

Keogh E, Ellery D, Hunt C, Hannent I (2001) Selective attentional bias for pain-related stimuli amongst pain fearful individuals. Pain 91:91-100.

King TE, Joynes RL, Meagher MW, Grau JW (1996) Impact of shock on pain reactivity. II. Evidence for enhanced pain. J Exp Psychol Anim Behav Proc 22:265-278.

Lang PJ, Davis M, Öhman A (2000) Fear and anxiety: animal models and human cognitive psychophysiology. J Affect Disord 61:137-159.

Lathe R (2001) Hormones and the hippocampus. J Endocrinol 169:205-231.

Lavenex P, Amaral DG (2000) Hippocampal-neocortical interaction: a hierarchy of associativity. Hippocampus 10:420-430.

Maier SF (1986) Stressor controllability and stress-induced analgesia. Ann NY Acad Sci 467:55-72.

McKenna JE, Melzack R (1992) Analgesia produced by lidocaine microinjection into the dentate gyrus. Pain 49:105-112.

Melzack R (1973) The puzzle of pain. New York: Basic Books.

Melzack R, Casey KL (1968) Sensory, motivational, and central control determinants of pain. In: The skin senses (Kenshalo DR, ed), pp 423-439. Springfield, IL: Thomas.

Miller SM (1981) Predictability and human stress: toward a clarification of evidence and theory. In: Advances in experimental social psychology, vol. 14 (Berkowitz L, ed), pp 203-256. New York: Academic.

Morris JS, Öhman A, Dolan RJ (1998) Conscious and unconscious emotional learning in the human amygdala. Nature 393:467-470.

Naliboff BD, Derbyshire SWG, Munakata J, Berman S, Mandelkern M, Chang L, Mayer EA (2001) Cerebral activation in patients with irritable bowel syndrome and control subjects during rectosigmoid stimulation. Psychosom Med 63:365-375.

Obrist PA (1981) Cardiovascular psychophysiology, a perspective. New York: Plenum.

O’Doherty J, Kringelbach ML, Rolls ET, Hornak J, Andrews C (2001) 
Abstract reward and punishment representations in the human orbitofrontal cortex. Nat Neurosci 4:95-102.

Pearse D, Mirza A, Leah J (2001) Jun, Fos and Krox in the hippocampus after noxious stimulation: simultaneous-input-dependent expression and nuclear speckling. Brain Res 894:193-208.

Petrovic P, Petersson KM, Ghatan PH, Stone-Elander S, Ingvar M (2000) Pain-related cerebral activation is altered by a distracting cognitive task. Pain 85:19-30.

Peyron R, García-Larrea L, Grégoire MC, Costes N, Convers P, Lavenne F, Mauguière F, Michel D, Laurent B (1999) Haemodynamic brain responses to acute pain in humans: sensory and attentional networks. Brain 122:1765-1779.

Peyron R, Laurent B, García-Larrea L (2000) Functional imaging of brain responses to pain. A review and meta-analysis. Neurophysiol Clin 30:263-288.

Ploghaus A, Tracey I, Gati JS, Clare S, Menon RS, Matthews PM, Rawlins JNP (1999) Dissociating pain from its anticipation in the human brain. Science 284:1979-1981.

Ploghaus A, Tracey I, Clare S, Gati JS, Rawlins JNP, Matthews PM (2000) Learning about pain: the neural substrate of the prediction error for aversive events. Proc Natl Acad Sci USA 97:9281-9286.

Porro CA, Cettolo V, Francescato MP, Baraldi P (1998) Temporal and intensity coding of pain in human cortex. J Neurophysiol 80:3312-3320.

Prado WA, Roberts MH (1985) An assessment of the antinociceptive and aversive effects of stimulating identified sites in the rat brain. Brain Res 340:219-228.

Price DD (1988) Psychological and neural mechanisms of pain. New York: Raven.

Rainville P, Duncan GH, Price DD, Carrier B, Bushnell MC (1997) Pain affect encoded in human anterior cingulate but not somatosensory cortex. Science 277:968-971.

Rauch SL, Savage CR, Alpert NM, Miguel EC, Baer L, Breiter HC, Fischman AJ, Manzo PA, Moretti C, Jenike MA (1995) A positron emission tomographic study of simple phobic symptom provocation. Arch Gen Psychiatry 52:20-28.

Reiman EM, Fusselman MJ, Fox PT, Raichle ME (1989) Neuroanatomical correlates of anticipatory anxiety. Science 243:1071-1074.

Rhudy JL, Meagher MW (2000) Fear and anxiety: divergent effects on human pain thresholds. Pain 84:65-75.

Sawamoto N, Honda M, Okada T, Hanakawa T, Kanda M, Fukuyama H, Konishi J, Shibasaki H (2000) Expectation of pain enhances responses to nonpainful somatosensory stimulation in the anterior cingulate cortex and parietal operculum/posterior insula: an event-related functional magnetic resonance imaging study. J Neurosci 20:7438-7445.

Schneider F, Habel U, Holthusen H, Kessler C, Posse S, Müller-Gärtner HW, Arndt JO (2001) Subjective ratings of pain correlate with subcortical-limbic blood flow: an fMRI study. Neuropsychobiology 43:175-185.
Schumacher R, Velden M (1984) Anxiety, pain experience, and pain report: a signal detection study. Percept Mot Skills 58:339-349.

Sinha R, Sharma R, Mathur R, Nayar U (1999) Hypothalamo-limbic involvement in modulation of tooth-pump stimulation evoked nociceptive response in rats. Indian J Physiol Pharmacol 43:323-331.

Sternbach RA (1968) Pain: a psychophysiological analysis. New York: Academic.

Suls J, Wan CK (1989) Effect of sensory and procedural information on coping with stressful medical procedures and pain: a meta-analysis. J Consult Clin Psychol 57:372-379.

Talairach J, Tournoux P (1988) Co-planar stereotaxic atlas of the human brain. Stuttgart, Germany: Thieme.

Tölle TR, Kaufmann T, Siessmeier T, Lautenbacher S, Berthele A, Munz F, Zieglgänsberger W, Willoch F, Schwaiger M, Conrad B, Bartenstein P (1999) Region-specific encoding of sensory and affective components of pain in the human brain: a positron emission tomography correlation analysis. Ann Neurol 45:40-47.

Tracey I, Becerra L, Chang I, Breiter H, Jenkins L, Borsook D, Gonzalez RG (2000) Noxious hot and cold stimulation produce common patterns of brain activation in humans: a functional magnetic resonance imaging study. Neurosci Lett 288:159-162.

Treede RD, Kenshalo DR, Gracely RH, Jones AK (1999) The cortical representation of pain. Pain 79:105-111.

Treede RD, Apkarian AV, Bromm B, Greenspan JD, Lenz FA (2000) Cortical representation of pain: functional characterization of nociceptive areas near the lateral sulcus. Pain 87:113-119.

van den Hout JH, Vlaeyen JW, Houben RM, Soeters AP, Peters ML (2001) The effects of failure feedback and pain-related fear on pain report, pain tolerance, and pain avoidance in chronic low back pain patients. Pain 92:247-257.

Van Hoesen GW (1995) Anatomy of the medial temporal lobe. Magn Reson Imaging 13:1047-1055.

Vogt BA, Derbyshire S, Jones AK (1996) Pain processing in four regions of human cingulate cortex localized with co-registered PET and MR imaging. Eur J Neurosci 8:1461-1473.

Wei F, Xu ZC, Qu Z, Milbrandt J, Zhuo M (2000) Role of EGR1 in hippocampal synaptic enhancement induced by tetanic stimulation and amputation. J Cell Biol 149:1325-1334.

Weisenberg M, Aviram O, Wolf Y, Raphaeli N (1984) Relevant and irrelevant anxiety in the reaction to pain. Pain 20:371-383.

Witter MP, Naber PA, van Haeften T, Machielsen WC, Rombouts SA, Barkhof F, Scheltens P, Lopes da Silva FH (2000) Corticohippocampal communication by way of parallel parahippocampalsubicular pathways. Hippocampus 10:398-410.

Woolrich M, Ripley B, Brady J, Smith S (2000) Nonparametric estimation of temporal autocorrelation in FMRI. NeuroImage 11:610.

Zheng F, Khanna S (1999) Morphine reversed formalin-induced CA1 pyramidal cell suppression via an effect on septohippocampal neural processing. Neuroscience 89:61-71. 